\title{
Short communication: Use of the BetaStar Plus assay for detection of ceftiofur antimicrobial residues in milk from individual cows following intramammary treatment for mastitis
}

\author{
D. L. Grooms, ${ }^{* 1}$ B. Norby, ${ }^{*}$ K. E. Grooms, ${ }^{*}$ E. N. Jagodzinski, ${ }^{*}$ R. J. Erskine, ${ }^{*}$ L. W. Halbert, ${ }^{*}$ J. F. Coetzee,† \\ L. Wulf, $†$ and J. A. Ricef \\ *Department of Large Animal Clinical Sciences, Michigan State University, East Lansing 48895 \\ †Department of Veterinary Diagnostic and Production Animal Medicine, lowa State University, Ames 50011 \\ $\ddagger$ Neogen Corporation, Lansing, MI 48912
}

\begin{abstract}
Development and use of on-farm assays to detect antimicrobial residues in milk is important to reduce the risk of violative residues in marketed milk. The objective of this study was to evaluate the effectiveness of a lateral-flow immunodiagnostic assay (BetaStar Plus, Neogen Corp., Lansing, MI) in detecting ceftiofur residues in milk from individual cows treated for mastitis. This assay is currently approved by the US Federal Drug Administration (FDA) for detecting $\beta$-lactam residues in commingled milk. Forty-five dairy cows with clinical mastitis from 4 dairy farms were enrolled and treated intramammary with $125 \mathrm{mg}$ of ceftiofur hydrochloride (Spectramast LC, Zoetis, Madison, NJ) according to the manufacturer's label recommendation. Composite milk samples were collected (A) before first intramammary antimicrobial treatment, (B) before the last intramammary antimicrobial treatment, (C) the last milking of the product-labeled milk withhold, (D) the first milking after the product-labeled milk withhold had been met, and (E) $72 \mathrm{~h}$ after the product-labeled milk withhold had been met. Samples were tested using the BetaStar Plus assay within $48 \mathrm{~h}$ of collection. Parallel samples were analyzed by liquid chromatography-tandem mass spectrometry (LC-MS/MS) and for somatic cell count and milk components. The BetaStar Plus assay identified $6.7,60.0,46.7,22.2$, and $6.7 \%$ positive samples at each of the respective time points. The assay had sensitivity and specificity of 100 and $84.7 \%$, respectively, compared with liquid chromatography-tandem mass spectrometry analysis using FDA published residue tolerance levels for ceftiofur (or ceftiofur metabolites) as a threshold. The BetaStar Plus assay could be useful
\end{abstract}

Received August 29, 2014.

Accepted June 2, 2015.

${ }^{1}$ Corresponding author: grooms@cvm.msu.edu for detecting ceftiofur residues in milk from individual cows following intramammary treatment for mastitis before the milk is shipped for processing.

Key words: milk, antibiotic, residue

\section{Short Communication}

Rapid assays for on-farm detection of antimicrobial residues in milk from individual cows are needed to reduce risks of violative residues in marketed milk. Currently, several assays are approved by the US Federal Drug Administration (FDA) and available for testing raw commingled milk from bulk storage tanks for antimicrobial residues as mandated by the Pasteurized Milk Ordinance. However, these tests were not originally designed to, nor are they approved by the FDA to, detect residues in milk from individual cows. The performance of these assays when used for testing milk from individual cows is often unknown. Knowing how residue detection assays developed for commingled milk perform when used in milk from individual cows may be valuable for incorporation into on-farm residue prevention programs.

The BetaStar Plus assay (Neogen Corporation, Lansing, MI) is a rapid, lateral-flow immunodiagnostic assay that is FDA approved for detecting $\beta$-lactam and cephalosporin antimicrobial residues in raw commingled milk (FDA, 2011a). The assay can detect $\beta$-lactam antimicrobial residues, including amoxicillin, ampicillin, cephapirin, cloxacillin, penicillin, and ceftiofur, at levels at or below FDA tolerance levels (Abouzied et al., 2012). The assay utilizes 2 detection systems: ceftiofur is detected by means of an anti-desfuroylceftiofur monoclonal antibody, whereas other $\beta$-lactams are detected with a $\beta$-lactam bacterial protein receptor (Abouzied et al., 2012). The objective of this study was to evaluate the BetaStar Plus assay for detecting ceftiofur (and its primary metabolites) residues in milk collected from individual dairy cows that were treated for mastitis using intramammary ceftiofur. 
Lactating dairy cows with clinical mastitis from 4 dairy farms located in central Michigan were enrolled in the study. All farms milked cows twice daily. Enrollment criteria included lactating cows with no previous treatment history for mastitis in the current lactation and mild to moderate clinical mastitis as determined by the appearance of abnormal milk, swelling of a quarter, or both. Cows that exhibited systemic signs of illness resulting from mastitis or parallel disease conditions, such as fever or diarrhea, were excluded. Cows were treated with $125 \mathrm{mg}$ of ceftiofur hydrochloride (Spectramast LC, Zoetis, Madison, NJ) by intramammary infusion for 2 to 8 consecutive days according to the manufacturer's label recommendation. Information collected on enrolled cows included identification, parity, days in milk, reproductive status (open, bred, pregnant), and date of the first treatment.

A 100-mL composite milk sample was collected from each enrolled cow by farm personnel at the following time points: (A) the milking immediately before first intramammary antimicrobial treatment, (B) the milking immediately before the last intramammary antimicrobial treatment, $(\mathrm{C})$ the last milking of the productlabeled milk withhold, (D) the first milking after the product-labeled milk withhold had been met, and (E) the first milking $72 \mathrm{~h}$ after the product-labeled milk withhold had been met. Samples were collected at various times in relationship to when the cows were treated to increase the likelihood of having samples containing varying levels of residues. Sample A was taken before treatment to represent milk most likely to have no residue in it. Under normal circumstances, this milk would not be tested either individually or as part of a bulk tank sample and is likely to be abnormal, which may affect screening test performance. Sample B was taken to represent samples that would have a high likelihood of having a violative residue in it. Samples C, D, and E represent samples more likely to be taken as part of an on-farm residue-screening program. Samples were collected into 4-ounce polypropylene screw-top containers following standard operating procedures for udder preparation used for each farm. Following collection, samples were refrigerated and then retrieved by study personnel within $24 \mathrm{~h}$ of collection and transported on ice to the laboratory where they were refrigerated until processing.

In the laboratory, each individual milk sample was thoroughly mixed by inverting 5 times; then, $25 \mathrm{~mL}$ of milk was allocated into 4 separate polypropylene snap-top containers. One subsample was tested using the BetaStar Plus assay. The second subsample was frozen at $-4^{\circ} \mathrm{C}$ and then submitted to the Iowa State University Pharmacology Analytical Support Team (PhAST, Iowa State University, Ames) for liquid chromatography-tandem mass spectrometry (LC-MS/ MS). The third subsample was frozen at $-4^{\circ} \mathrm{C}$ as a backup sample. A fourth $25-\mathrm{mL}$ subsample was allocated into a polypropylene snap-top vial containing the preservative bronopol and sent to an outside laboratory (Universal Labs Services, Lansing, MI) for milk SCC and component analysis. In addition, a sample of the aliquot used for the BetaStar Plus assay was tested using the California Mastitis Test (CMT; Jorgensen Laboratories, Loveland, CO).

Milk samples were analyzed by using the BetaStar Plus assay at Michigan State University (Grooms Laboratory) on unfrozen samples within $48 \mathrm{~h}$ of collection using the manufacturer's recommended procedures. The BetaStar Plus assay is a receptor- and antibodybased lateral-flow assay. The primary metabolite of ceftiofur, desfuroylceftiofur, is detected by an anti-desfuroylceftiofur monoclonal antibody. Other $\beta$-lactams (including parent ceftiofur) are detected by binding with a $\beta$-lactam bacterial receptor protein. The result of the assay is a "sample-to-negative control" ratio determined after inserting the lateral-flow device into a photometric reader (Accuscan III, Neogen Corp.). The photometric reader measures the relative intensity of the test and control lines. The sample-to-negative control was automatically calculated by the reader and the results recorded. A sample-to-negative control ratio of $\leq 1.0$ was considered positive, whereas a ratio $>1.0$ was considered negative.

Frozen samples were banked and submitted to PhAST for LC-MS/MS. Samples were screened initially using a multidrug ( $\sim 50$ antibiotics) residue panel that included screening for ceftiofur and ceftiofur metabolites (hereafter referred to as "screening" LC-MS/MS) as well as other antimicrobial drugs. Milk samples for this screening panel were extracted into acetonitrile by a QuChERS procedure (Aguilera-Luiz et al., 2008; Clark et al., 2011) and cleaned up by solid-phase extraction on Strata-X (Phenomenex, Torrance, CA). Any sample in which ceftiofur or ceftiofur metabolite residues was detected was then rerun using an LC-MS/MS protocol (hereafter referred to as "definitive" LC-MS/MS) specific for only ceftiofur. In this protocol, all forms of ceftiofur (i.e., parent, metabolites, and protein-bound residues) were cleaved to desfuroylceftiofur (DFC) and then derivatized to desfuroylceftiofur acetamide (DCA; Hornish et al., 2003). Each set of milk samples was analyzed concurrently with 9 calibrators (1-500 ng/mL) and 3 quality control samples. An internal standard was added to all samples before cleavage, derivatization, and solid-phase extraction cleanup on Oasis HLB cartridges (Waters, Milford, MA).

The screening LC-MS/MS analysis was performed on a TSQ Discovery Max triple quadrupole mass spec- 
trometer (Thermo Scientific, San Jose, CA) coupled to a Surveyor MS pump and Surveyor autosampler. The definitive LC-MS/MS analysis was performed on an LTQ ion trap mass spectrometer (Thermo Scientific) coupled to an Agilent 1100 binary pump and autosampler (Agilent, Santa Clara, CA). The screening and definitive LC-MS/MS analysis separations were performed on an Kinetex XB-C18 column $(150 \times 2.1$ $\mathrm{mm}$ i.d., $2.6 \mu \mathrm{m}$, Phenomenex) and an ACE $3 \mathrm{C} 18$ column $(150 \times 2.1 \mathrm{~mm}$ i.d., MAC-MOD Analytical, Chads Ford, PA), respectively. Mobile phases A and $\mathrm{B}$ were $0.1 \%$ formic acid in LC-MS grade water and acetonitrile, respectively. Both LC-MS/MS analysis used positive electrospray ionization $\left(\mathrm{ESI}^{+}\right)$. TOXID software (Thermo Fisher Scientific Inc., Waltham, MA) was used for detection of antibiotics in screening samples. Samples containing antibiotics were then further processed through the quantitation software of the Xcalibur software (Thermo Fisher Scientific) to arrive at quantitative analytical results. The definitive analysis used full-scan MS fragmentation of the pseudomolecular parent ion of DCA at a mass-to-charge ratio $(\mathrm{m} / \mathrm{z})$ of 487 , which fragmented to 5 ions that were used for quantitation of DCA at $m / z$ of $166,197,241$, 324 , and 396.

To determine the effect of milk freezing on the results of the BetaStar Plus assay and LC-MS/MS, milk samples were collected from 4 individual cows with mastitis $24 \mathrm{~h}$ after intramammary infusion of $125 \mathrm{mg}$ of ceftiofur hydrochloride (Spectramast LC). Each sample was then subdivided into eight $15-\mathrm{mL}$ aliquots and refrigerated at $4^{\circ} \mathrm{C}$. Four of the aliquots were shipped overnight to PhAST. Once samples had arrived at PhAST, one unfrozen aliquot was tested by the BetaStar Plus assay and LC-MS/MS in parallel by the respective laboratories (time $t=0 \mathrm{~h}$ ). The remaining aliquots were frozen and stored at $-20^{\circ} \mathrm{C}$. Then at $t=24 \mathrm{~h}, t=7 \mathrm{~d}$, and $t=28 \mathrm{~d}$, an aliquot of each sample was thawed by placing in a circulating water bath at $35^{\circ} \mathrm{C}$ and then subsequently tested using the BetaStar Plus assay and LC-MS/MS by the respective laboratories.

The BetaStar Plus assay results were interpreted according to the manufacturers recommendations. In brief, a sample-to-negative control ratio of $\leq 1.0$ on either the $\beta$-lactam or ceftiofur indicator line on the BetaStar Plus assay was considered positive, whereas a ratio $>1.0$ on both lines was considered negative. The quantity of the 5 DCA ions detected by LC-MS/MS screening was summed to give a total ceftiofur equivalent in parts per billion. If the sum was $\geq 100 \mathrm{ppb}$, the sample was considered to be above the FDA violative residue level and therefore classified as positive for ceftiofur. If it was $<100 \mathrm{ppb}$, the sample was considered below the violative residue level, and therefore classi- fied as negative for ceftiofur. For the definitive test, the cutoff for a positive result was also $100 \mathrm{ppb}$. Data on test results, CMT, and milk components were entered into Excel (Microsoft Corp., Redmond, WA). Data management and statistical analyses were conducted using Stata 13.1 (Stata Corp., College Station, TX). The proportion of positive tests (BetaStar Plus assay, LC-MS/MS screening test, and LC-MS/MS definitive test) was calculated for each sampling point and across all sampling points. The sensitivities and specificities of the BetaStar Plus assay test were calculated using both the screening and definitive test results as reference tests, respectively. The sensitivity was calculated as the conditional probability of a positive test result if the reference test was positive $[P(\mathrm{~T}+\mid \mathrm{D}+)]$, and the specificity was calculated as the probability of a negative test result if the reference test was negative $[P(\mathrm{~T}-\mid \mathrm{D}-)]$, where $P$ indicates probability, $\mathrm{T}(+/-)$ is the positive or negative outcome on the BetaStar Plus, and D $(+/-)$ is the positive or negative outcome on either of the reference tests. The $95 \%$ confidence intervals for the proportion of positive samples and sensitivities and specificities were calculated using the exact method (Stata, 2013).

Data from sampling points B, C, D, and E were used to estimate potential associations between CMT score, SCC, fat, lactose, protein and solids, and BetaStar Plus assay test results using logistic regression with animals as a random effect. To assess if continuous variables, or $\log _{10}$-transformation of continuous variables, were linear in the logit, the continuous variables were first divided into 4 categories so that each category had the same approximate number of observations $(\mathrm{n}=35-41$ per category). In the case of SCC, samples were divided into 5 categories that correspond to the commonly used CMT categories (McFaddin, 2011). Second, linearity in the logit was determined by visually assessing the linearity of a graph with the logit plotted as a function of the mid-point of the categories of each continuous variable (Hosmer et al., 2013). If the plot did not show a linear relationship in the logit for a categorical variable, the original continuous variable was $\log _{10}$-transformed and the second step in the process described above was repeated. If a categorized continuous variable or a $\log _{10^{-}}$ transformed continuous variable was not linear in the logit, the categorical variable was use in the model. Length of treatment was not included in the model because data from the manufacturer's New Animal Drug Application (NADA 141-238) for Spectramast LC Sterile Suspension (ceftiofur hydrochloride) indicates no difference in expected concentrations of ceftiofur in milk with variable therapy lengths (FDA-CVM, 2005).

The Friedman test, a nonparametric alternative to one-way ANOVA with repeated measures (Gibbons 
1985), was used to detect differences in ceftiofur equivalents and the BetaStar Plus assay sample-to-negative control photometric ratios in the same samples over time following freezing. A chi-squared test was used to compare the frequency of samples by $\log _{10}(\mathrm{SCC})$ category and sampling time.

In total, 225 individual milk samples from 45 cows were tested with both the BetaStar Plus assay and LC-MS/MS. The BetaStar Plus assay identified 6.7, $60.0,46.7,22.2$, and $6.7 \%$ positive samples at each of the respective time points (Table 1). Overall, across all sampling points, the assay had sensitivity and specificity of $100 \%(\mathrm{CI}=90.0-100.0)$ and $84.7 \%(\mathrm{CI}=$ 78.8-89.5), respectively, compared with LC-MS/MS definitive ceftiofur analysis and using the FDA published residue tolerance level of $100 \mathrm{ppb}$ (Table 2). When calculated using the screening LC-MS/MS results and setting a positive sample cutoff at $100 \mathrm{ppb}$, the sensitivity was $100 \%(\mathrm{CI}=76.8-100)$ and the specificity $76.3 \%$ (CI $=70.0-81.9$ ). The majority of samples that were positive on the BetaStar Plus assay but did not have ceftiofur residues above the FDA tolerance level of $100 \mathrm{ppb}$ (as determined by the definitive LC-MS/ MS) were found to have ceftiofur residues in the range of 10-100 ppb (Figure 1). Only 3 samples were positive by BetaStar Plus assay in which no ceftiofur residue could be detected by LC-MS/MS. All of these samples were from the A sampling point (before treatment with ceftiofur). Based on visual assessment of linearity of the logit plotted as a function of the midpoint categories of continuous variables, only 2 variables, $\log _{10}$ fat and protein, appeared linear in the logit. Hence, we elected to use the categorized continuous variable for all variables as described in the methods section. Samples with an increasing CMT score $(P=0.007)$ were more likely to be positive on the BetaStar Plus assay (Table 3). However, $\log _{10}(\mathrm{SCC})$ was not significantly $(P=0.24)$ associated with outcome of the BetaStar Plus assay.
The reason for this discrepancy is not clear, because in general, CMT and SCC are correlated (Luedecke et al., 1967). One possible reason is that the CMT is a subjective assay that relies on visual interpretation. Although laboratory personnel were trained in CMT interpretation, the test requires a judgment to be made, which can be subjective. Conversely, SCC is conducted using an automated cell counter, which eliminates subjectivity. In addition, most correlations between CMT and SCC have been done in cows with subclinical mastitis, whereas our study was done in cows with clinical mastitis (Luedecke et al., 1967; Sargeant et al., 2001). For the $\log _{10}(\mathrm{SCC})$ analysis, we divided samples into quartiles that correlate with CMT scores (Ruegg 2005). Thirtyeight $(25 \%)$ cows had a $\log _{10}(\mathrm{SCC}) \geq 7.4$, with $7,9,8$, and 14 of these cows sampled during periods B, C, D, and $\mathrm{E}$, respectively. In general, the SCC were very high for cows at all sampling points in this study. The median $\log _{10}(\mathrm{SCC})$ was 6.6 , and 14 cows with a $\log _{10}(\mathrm{SCC})$ $\geq 7.4$ in cows $72 \mathrm{~h}$ after treatment was unexpectedly high. We expected that cows with mastitis would have high SCC but also that most cows would have cleared their mastitis infection $72 \mathrm{~h}$ after the last treatment. However, previous studies have demonstrated that SCC in treated cows does not always return to normal levels $(<200,000$ cells $/ \mathrm{mL})$ and can be affected by variables such as the causative pathogen and previous history of clinical mastitis (Pinzón-Sánchez and Ruegg, 2011). The SCC in a sample might affect the outcome of the BetaStar Plus assay; however, in the current study, it is unlikely that this would have affected the results because the frequency of samples in the different SCC categories did not differ by sampling time points $(P$ $=0.31$ ). Nonetheless, caution should be applied when extrapolating our results for SCC directly to a situation where the SCC are lower. We found no association between percentage lactose, solids, protein or $\log _{10}$ fat and the odds of being positive on the BetaStar Plus assay.

Table 1. Point estimate of proportions of positive samples (95\% CI in parentheses, unless otherwise noted) across all time points as determined by the BetaStar Plus assay (Neogen Corp., Lansing, MI) and the screening and definitive liquid chromatography-tandem MS (LC-MS/MS) using a ceftiofur equivalent of $\geq 100 \mathrm{ppb}$ as the positive cut off

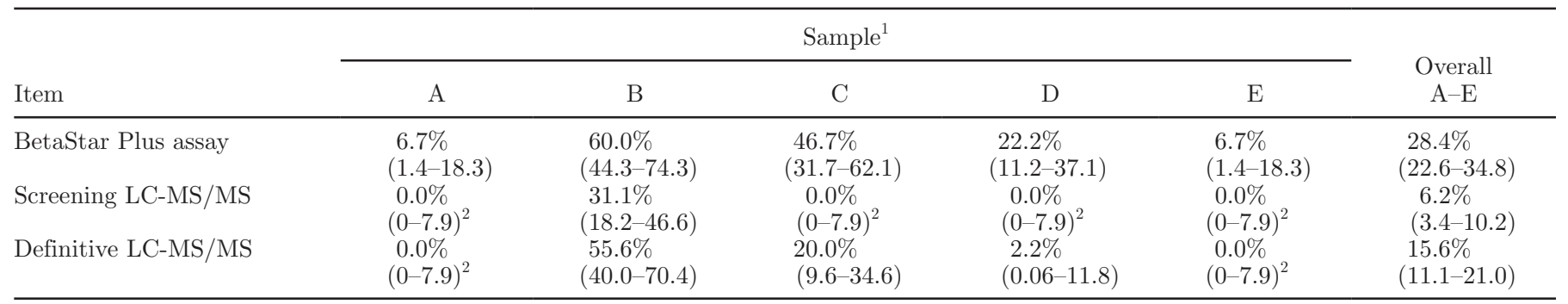

${ }^{1}$ Samples: $\mathrm{A}=$ before first intramammary antimicrobial treatment, $\mathrm{B}=$ before the last intramammary antimicrobial treatment, $\mathrm{C}=$ the last milking of the product-labeled milk withhold, $\mathrm{D}=$ the first milking after the product-labeled milk withhold had been met, and $\mathrm{E}=72 \mathrm{~h}$ after the product-labeled milk withhold had been met.

${ }^{2} 97.5 \%$ CI. 
GROOMS ET AL.

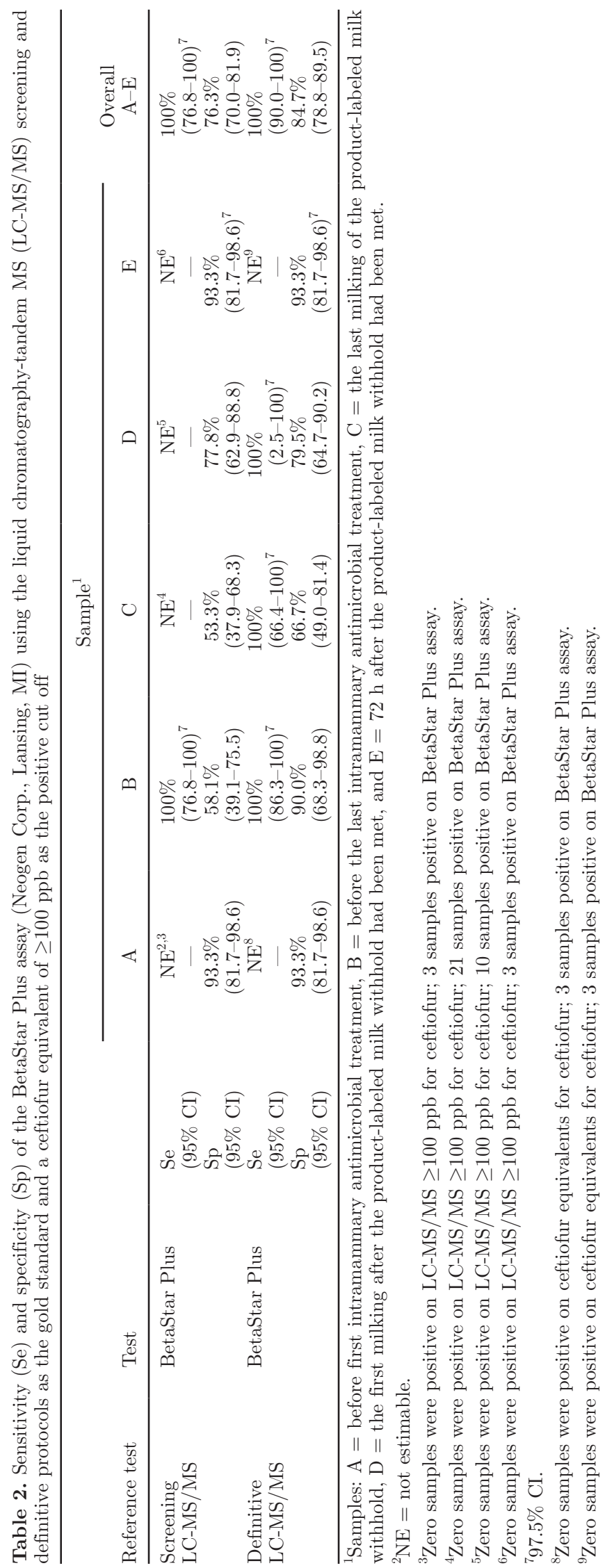




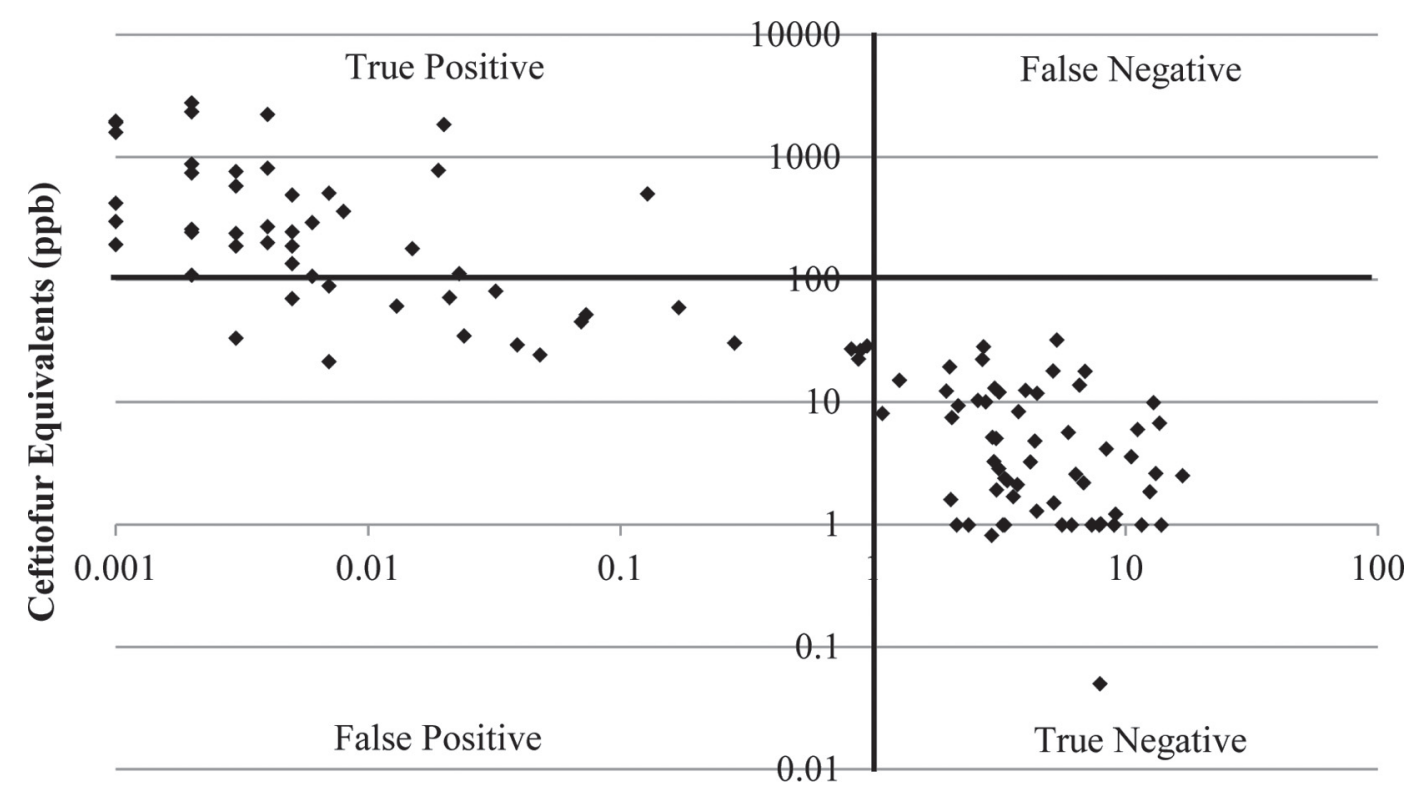

BetaStar Plus Sample-to-Negative Control Ratio

Figure 1. Relationship of ceftiofur equivalents (parts per billion, ppb) as determined by liquid chromatography-tandem MS (LC-MS/MS) to the BetaStar Plus assay (Neogen Corp., Lansing, MI) sample-to-negative control photometric ratio in milk samples collected from individual lactating cows treated with intramammary ceftiofur. The Food and Drug Administration tolerance level for ceftiofur in milk is 100 ppb (bold horizontal line). A sample was considered positive on the BetaStar Plus assay when the sample-to-negative control ratio was $\leq 1.0$ (bold vertical line). Each quartile is labeled as true positive, false positive, and so on, based on the result of the LC-MS/MS being the gold standard or "correct" result.

All samples used in the stability study remained positive by the BetaStar Plus assay following freezing for up to $28 \mathrm{~d}$. We detected no significant change in ceftiofur equivalents detected by LC-MS/MS $(P=0.55)$ or the BetaStar Plus assay sample-to-negative control photometric ratio $(P=0.17)$ in samples frozen up to $28 \mathrm{~d}$.

The BetaStar Plus assay was originally designed and is approved by FDA to detect $\beta$-lactam antimicrobial residues in commingled bulk tank milk at levels specified by the FDA Pasteurized Milk Ordinance (FDA, 2011a). Our results demonstrate that the assay can also accurately detect $\beta$-lactam antimicrobial residues, specifically ceftiofur, in individual cow milk samples, thus making it useful for residue screening before commingling of individual cow milk.

Overall and more specifically, at the first milking after the product-labeled milk withhold had been met (sample D), the sensitivity and specificity of the BetaStar Plus assay were similar to that reported for other residue detection assays when used on individual cow milk samples (Seymour et al., 1988; Van Eenennaam et al., 1993; Andrew et al., 1997; Hillerton et al., 1999; Gibbons-Burgener et al., 2001). In our study, the sensitivity of the BetaStar Plus assay was high. We found no samples with violative ceftiofur residues by LC-MS/MS that were not detected by the BetaStar Plus assay. The ability of the assay to accurately detect the presence of violative residues is important from a food safety perspective, as well as from the perspective of producer and consumer confidence. Conversely, the specificity of the assay was lower, resulting in multiple false-positive results, a finding that has been reported with respect to other antimicrobial screening assays (Van Eenennaam et al., 1993; Andrew et al., 1997; Gibbons-Burgener et al., 2001). There are several possible explanations for this finding. First, it should be noted that the BetaStar Plus assay is capable of detecting ceftiofur residues below the FDA tolerance level of $100 \mathrm{ppb}$. In validation studies, $60 \%$ of milk samples containing 65 ppb of ceftiofur were positive on the BetaStar Plus assay (Abouzied et al., 2012). The need to have greater analytical sensitivity is necessary to ensure near $100 \%$ sensitivity for detecting residues at or above the FDA tolerance level. This is confirmed visually in Figure 1 where the majority of false-positive samples had levels of ceftiofur in the 60 to $100 \mathrm{ppb}$ range.

Another factor that may decrease performance of the assay is increased variability of individual cow milk samples compared with commingled milk samples. Previous studies have demonstrated that increased milk fat or protein increased the likelihood of false-positive 
Table 3. Association between BetaStar Plus assay (Neogen Corp., Lansing, MI) outcome and California Mastitis Test (CMT), SCC, and percentage milk protein, fat, nonfat solids, and lactose across all sampling points except sample $\mathrm{A}^{1}$

\begin{tabular}{|c|c|c|c|c|c|}
\hline Variable & $\begin{array}{c}\text { No. of } \\
\text { samples }\end{array}$ & $\begin{array}{l}\text { Coefficient } \\
\text { (odds ratio) }\end{array}$ & $\begin{array}{c}\mathrm{SE} \\
\text { (odds ratio } \mathrm{SE} \text { ) }\end{array}$ & $P$-value & $\begin{array}{l}\text { Overall } \\
P \text {-value }\end{array}$ \\
\hline CMT & & & & & 0.007 \\
\hline 0 & 57 & Reference & & & \\
\hline 1 & 88 & $1.47(4.36)$ & $0.51(2.23)$ & 0.004 & \\
\hline 2 & 24 & $1.88(6.56)$ & $0.67(4.42)$ & 0.005 & \\
\hline 3 & 8 & $2.50(12.12)$ & $1.01(12.21)$ & 0.013 & \\
\hline $\operatorname{SCC}\left(\times 10^{3}\right)$ & & & & & 0.24 \\
\hline$<200$ & 27 & Reference & & & \\
\hline$\geq 200<400$ & 21 & $-0.16(0.85)$ & $0.82(0.70)$ & 0.844 & \\
\hline$\geq 400<1,200$ & 54 & $0.96(2.62)$ & $0.66(1.72)$ & 0.143 & \\
\hline$\geq 1,200<5,000$ & 43 & $1.07(2.90)$ & $0.68(1.98)$ & 0.118 & \\
\hline$\geq 5,000$ & 8 & $1.06(1.11)$ & $1.12(1.24)$ & 0.925 & \\
\hline Fat $\left(\log _{10}\right)$ & & & & & 0.95 \\
\hline$<0.8$ & 39 & Reference & & & \\
\hline$\geq 0.8<1.4$ & 38 & $-0.16(0.85)$ & $0.56(0.47)$ & 0.772 & \\
\hline$\geq 1.4<1.9$ & 38 & $-0.13(0.88)$ & $0.58(0.51)$ & 0.820 & \\
\hline$\geq 1.9$ & 38 & $-0.33(0.42)$ & $0.58(0.42)$ & 0.570 & \\
\hline Lactose & & & & & 0.84 \\
\hline$<3.7$ & 39 & Reference & & & \\
\hline$\geq 3.7<4.3$ & 40 & $0.47(1.61)$ & $0.58(0.94)$ & 0.416 & \\
\hline$\geq 4.3<4.6$ & 36 & $0.52(1.69)$ & $0.64(1.09)$ & 0.418 & \\
\hline$\geq 4.6$ & 38 & $0.36(1.44)$ & $0.66(0.96)$ & 0.583 & \\
\hline Nonfat solids & & & & & 0.53 \\
\hline$<4.5$ & 39 & Reference & & & \\
\hline$\geq 4.5<5.1$ & 40 & $0.88(2.40)$ & $0.60(1.44)$ & 0.145 & \\
\hline$\geq 5.1<5.5$ & 36 & $0.67(1.94)$ & $0.65(1.27)$ & 0.309 & \\
\hline$\geq 5.5$ & 38 & $0.46(1.59)$ & $0.67(1.07)$ & 0.69 & \\
\hline Protein & & & & & 0.15 \\
\hline$<2.7$ & 38 & Reference & & & \\
\hline$\geq 2.7<3.0$ & 41 & $-0.06(0.93)$ & $0.60(0.56)$ & 0.878 & \\
\hline$\geq 3.0<3.3$ & 35 & $0.34(1.41)$ & $0.63(0.89)$ & 0.635 & \\
\hline$\overline{\geq} 3.3$ & 38 & $1.18(3.26)$ & $0.62(2.02)$ & 0.065 & \\
\hline
\end{tabular}

${ }^{1}$ All variables except CMT and SCC were categorized into quartiles (labeled as $0,1,2,3$ ) so each quartile had approximately the same number of observations.

test results, depending on the assay used (Andrew et al., 1997; Andrew, 2000). In our study, milk samples with higher SCC or CMT scores were more likely to be positive on the BetaStar Plus assay. Intuitively, this makes sense because these high SCC or CMT samples more likely came from cows early in the treatment of their mastitis, and therefore were more likely to have residues in their milk. However, we found no association with any of the other milk components and the likelihood of being positive on the BetaStar Plus assay.

We found that 1 of $45(2.2 \%)$ samples had violative ceftiofur residues at the first milking following the withdrawal period (sample D) as determined by LC-MS/MS. This sample was positive on the BetaStar Plus assay as well. It is possible that this sample was collected early and in fact represented milk from before the labeled withdrawal time had been met. Alternatively, the detected residue may have been a result of the cow not being able to clear the ceftiofur or ceftiofur metabolites (DFC) effectively because of the unhealthy state of the udder. The withdrawal times of antimicrobials in cattle are established using healthy animals and may not represent withdrawal times that occur in diseased animals (FDA, 2011b). Additionally, other variables could factor into this finding, such as the amount of milk produced by the cow. Regardless of the cause, this demonstrates why using a screening test on milk that is believed to have been collected after the milk withdrawal time has been met can be useful in reducing the risk of accidentally introducing antimicrobial residues into the bulk tank and risking a positive bulk tank violative residue test. Even more useful would be the use of screening tests on individual cows when the risk of a violative residue may be higher such as when questions arise about uncertain drug administration or cases of mistaken cow identity. In situations such as these, the risk of antimicrobial residue issues is much more likely.

The study design and logistics of conducting LC-MS/ MS led to freezing of milk samples at $-20^{\circ} \mathrm{C}$ and then running them as batches later. An immediate concern was the stability of ceftiofur metabolites in frozen milk over time and how this would affect the LC-MS/MS results. Because of this, we conducted a small study to 
determine if metabolites of ceftiofur remained detectable in milk frozen at $4^{\circ} \mathrm{C}$ for up to $28 \mathrm{~d}$. Results from our study showed no significant change in the ceftiofur equivalents detected in frozen samples stored for up to $28 \mathrm{~d}$. In parallel, we tested the same frozen samples on the BetaStar Plus assay and all samples remained positive until $28 \mathrm{~d}$. This adds to previous data that demonstrated the assay could detect other $\beta$-lactam antimicrobials (penicillin and cephapirin) in frozen milk (Abouzied et al., 2012).

In summary, the BetaStar Plus assay is a useful tool for detecting ceftiofur antimicrobial residues in milk obtained from individual cows following intramammary treatment for mastitis and after the manufacturer's label-specified milk withdrawal period had been met. The sensitivity and specificity of the assay is similar to that reported for other tests used to detect antimicrobial residues in milk from individual cows. The high sensitivity of the BetaStar Plus assay for detecting $\beta$-lactam antimicrobial residues would significantly reduce the risk of commingling and marketing milk with violative $\beta$-lactam residues.

\section{REFERENCES}

Abouzied, M., D. Driksna, C. Walsh, M. Sarzynski, A. Walsh, D. Ankrapp, F. Klein, J. Rice, and M. Mozola. 2012. Validation study of the BetaStar plus lateral flow assay for detection of beta-lactam antibiotics in milk. J. AOAC Int. 95:1211-1221.

Aguilera-Luiz, M. M., J. L. Martínez Vidal, R. Romero-González, and A. G. Frenich. 2008. Multi-residue determination of veterinary drugs in milk by ultra-high-pressure liquid chromatography-tandem mass spectrometry. J. Chromatogr. A 1205:10-16.

Andrew, S. M. 2000. Effect of fat and protein content of milk from individual cows on the specificity rates of antibiotic residue screening tests. J. Dairy Sci. 83:2992-2997.

Andrew, S. M., R. A. Frobish, M. J. Paape, and L. J. Maturin. 1997. Evaluation of selected antibiotic residue screening tests for milk from individual cows and examination of factors that affect the probability of false-positive outcomes. J. Dairy Sci. 80:3050-3057.

Clark, S. B., J. M. Storey, and S. B. Turnspeed. 2011. Optimization and validation of a multiclass screening and confirmation method for drug residues in milk using high-performance liquid chromatography/tandem mass spectrometry. J. AOAC Int. 94:383-393.

FDA (Food and Drug Administration). 2011a. M-I-11-3: Neogen Corporation BetaStar ${ }^{\circledR}$ Plus Beta-Lactam Assay and BetaStar ${ }^{\circledR}$ US Beta-Lactam Test. Accessed Jun. 18, 2014. http://www.fda.gov/Food/GuidanceRegulation/ GuidanceDocumentsRegulatoryInformation/Milk/ucm259267. htm.
FDA (Food and Drug Administration). 2011b. VICH GL46: Studies to evaluate the metabolism and residue kinetics of Veterinary drugs in food-producing animals: Metabolism study to determine the quantity and identify the nature of residues. Accessed Jun. 30, 2014. http://www.fda.gov/downloads/AnimalVeterinary/ GuidanceComplianceEnforcement/GuidanceforIndustry/ UCM207939.pdf.

FDA-CVM (Food and Drug Administration-Center for Veterinary Medicine). 2005. Freedom of Information Summary Original New Animal Drug Application NADA 141-238 SPECTRAMAST LC Sterile Suspension (ceftiofur hydrochloride). Accessed Feb. 9, 2015. http://www.fda.gov/downloads/AnimalVeterinary/ Products/ApprovedAnimalDrugProducts/FOIADrugSummaries/ ucm118051.pdf.

Gibbons, J. D. 1985. Nonparametric Statistical Inference. 2nd ed. Marcel Dekker, New York, NY.

Gibbons-Burgener, S. N., J. B. Kaneene, J. W. Lloyd, J. F. Leykam, and R. J. Erskine. 2001. Reliability of three bulk-tank antimicrobial residue detection assays used to test individual milk samples from cows with mild clinical mastitis. Am. J. Vet. Res. 62:17161720.

Hillerton, J. E., B. I. Halley, P. Neaves, and M. D. Rose. 1999. Detection of antimicrobial substances in individual cow and quarter milk samples using Delvotest microbial inhibitor tests. J. Dairy Sci. 82:704-711.

Hornish, R. E., P. J. Hamlow, and S. A. Brown. 2003. Multilaboratory trial for determination of ceftiofur residues in bovine and swine kidney and muscle, and bovine milk. J. AOAC Int. 86:30-38.

Hosmer, D. W., S. Lemeshow, and R. X. Sturdivant. 2013. Applied Logistic Regression. 3rd ed. John Wiley and Sons, Hoboken, NJ.

Luedecke, L. O., T. L. Forster, and U. S. Ashworth. 1967. Relationship between California Mastitis Test reaction and leucocyte count, catalase activity, and A-esterase activity of milk from opposite quarters. J. Dairy Sci. 50:1592-1596.

McFaddin, M. 2011. California Mastitis Test and Milk Quality. Michigan Dairy Review. Accessed Jun. 1, 2015. http://dairyteam.msu. edu/uploads/files/cmt.pdf.

Pinzón-Sánchez, C., and P. L. Ruegg. 2011. Risk factors associated with short-term post-treatment outcomes of clinical mastitis. J. Dairy Sci. 94:3397-3410.

Ruegg, P. L. 2005. California Mastitis Test (CMT) Fact Sheet 1. Accessed May 2, 2015. http://milkquality.wisc.edu/wp-content/ uploads/2011/09/california-mastitis-test-fact-sheet.pdf

Sargeant, J. M., K. E. Leslie, J. E. Shirley, B. J. Pulkrabek, and G. H. Lim. 2001. Sensitivity and specificity of somatic cell count and California Mastitis Test for identifying intramammary infection in early lactation. J. Dairy Sci. 84:2018-2024.

Seymour, E. H., G. M. Jones, and M. L. McGilliard. 1988. Comparisons of on-farm screening tests for detection of antibiotic residues. J. Dairy Sci. 71:539-544.

Stata. 2013. Confidence intervals for means, proportions, and counts. Pages 262-273 in Stata Base Reference Manual, Release 13. Stata Press, College Station, TX.

Van Eenennaam, A. L., J. S. Cullor, L. Perani, I. A. Gardner, W. L. Smith, J. Dellinger, and W. M. Guterbock. 1993. Evaluation of milk antibiotic residue screening tests in cattle with naturally occurring clinical mastitis. J. Dairy Sci. 76:3041-3053. 